\title{
Ascent Trajectory Optimisation for a Single-Stage-to-Orbit Vehicle with Hybrid Propulsion
}

\author{
Fabrizio Pescetelli, Edmondo Minisci $\dagger$ Christie Maddock $\ddagger$ Ian Taylor ${ }^{\dagger}$, Richard E Brown $^{\S}$ \\ Centre for Future Air-Space Transportation Technology, Dept. of Mechanical \& Aerospace Engineering \\ University of Strathclyde, Glasgow G4 OLT, Scotland, UK
}

This paper addresses the design of ascent trajectories for a hybrid-engine, high performance, unmanned, single-stage-to-orbit vehicle for payload deployment into low Earth orbit. A hybrid optimisation technique that couples a population-based, stochastic algorithm with a deterministic, gradient-based technique is used to maximize the final vehicle mass in low Earth orbit after accounting for operational constraints on the dynamic pressure, Mach number and maximum axial and normal accelerations. The control search space is first explored by the population-based algorithm, which uses a single shooting method to evaluate the performance of candidate solutions. The resultant optimal control law and corresponding trajectory are then further refined by a direct collocation method based on finite elements in time. Two distinct operational phases, one using an air-breathing propulsion mode and the second using rocket propulsion, are considered. The presence of uncertainties in the atmospheric and vehicle aerodynamic models are considered in order to quantify their effect on the performance of the vehicle. Firstly, the deterministic optimal control law is re-integrated after introducing uncertainties into the models. The proximity of the final solutions to the target states are analysed statistically. A second analysis is then performed, aimed at determining the best performance of the vehicle when these uncertainties are included directly in the optimisation. The statistical analysis of the results obtained are summarized by an expectancy curve which represents the probable vehicle performance as a function of the uncertain system parameters. This analysis can be used during the preliminary phase of design to yield valuable insights into the robustness of the performance of the vehicle to uncertainties in the specification of its parameters.

\section{Nomenclature}

$\begin{array}{llll}a & \text { Acceleration, } \mathrm{m} / \mathrm{s}^{2} \text { or speed of sound, } \mathrm{m} / \mathrm{s} & L & \text { Lift, } \mathrm{N} \\ A & \text { Area, } \mathrm{m}^{2} & m & \text { Mass, } \mathrm{kg} \\ c & \text { Specific heat capacity, } \mathrm{J} / \mathrm{K} \cdot \mathrm{kg} & M & \text { Mach number } \\ \mathbf{c} & \text { Control design vector } & p & \text { Probability, } \% \\ C & \text { Optimisation constraint } & P & \text { Pressure, Pa } \\ & \text { or aerodynamic coefficient } & q & \text { Dynamic pressure, Pa } \\ D & \text { Drag, } \mathrm{N} & Q_{r} & \text { Heating value of the fuel, } \mathrm{J} / \mathrm{kg} \\ E A S & \text { Equivalent air speed, } \mathrm{m} / \mathrm{s} & R & \text { Radius, } \mathrm{m} \\ f_{a} & \text { Fuel-air ratio } & S_{E} & \text { Sampling surface } \\ F & \text { Force or thrust, } \mathrm{N} & T & \text { Temperature, } \mathrm{K} \\ g & \text { Gravitation acceleration, } \mathrm{m} / \mathrm{s}^{2} & u_{b} & \text { Upper bound percentage, } \% \\ h & \text { Altitude above mean sea level, } \mathrm{m} \text { or km } & v & \text { Velocity, m/s } \\ I_{s p} & \text { Specific impulse, } \mathrm{s} & \alpha & \text { Angle of attack, rad } \\ l_{b} & \text { Lower bound percentage, } \% & \gamma & \text { Flight path angle, rad } \\ \end{array}$

\footnotetext{
*Postgraduate Research student

${ }^{\dagger}$ Lecturer

$\ddagger$ Research Fellow, AIAA Member

$\S$ Professor, AIAA Senior Member
} 


$\begin{array}{ll}\gamma_{i} & \text { Isentropic index } \\ \delta_{T} & \text { Throttle control } \\ \epsilon & \text { Angle between the thrust vector and } \\ & \text { velocity vector of the vehicle, rad } \\ \varepsilon & \text { Uncertainty distribution function } \\ \eta & \text { Efficiency, \% } \\ \theta & \text { Longitude, rad or deg E }\end{array}$

Subscript

$\begin{array}{ll}0 & \text { Initial time, usually } t=0 \\ \text { aero } & \text { Aerodynamic model } \\ \text { atm } & \text { Atmospheric model } \\ c & \text { Cut-off value } \\ \text { comb } & \text { Combustion } \\ D & \text { Drag } \\ e & \text { Exit of the engine } \\ E & \text { Earth } \\ f & \text { Final time } \\ h s & \text { Hypersonic }\end{array}$

$\begin{array}{ll}\lambda & \text { Latitude, rad or deg } \mathrm{N} \\ \mu & \text { Bank angle, rad } \\ \rho & \text { Density, } \mathrm{kg} / \mathrm{m}^{3} \\ \chi & \text { Path direction angle, rad } \\ \omega & \text { Angular velocity, rad } / \mathrm{s}\end{array}$

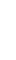


is also evaluated. While the analysis presented here is by no means comprehensive, the idea is to conduct a preliminary assessment of the performance of the vehicle in the presence of uncertainties in its modeling and characterization.

Two different approaches are used. In the first approach, a set of trajectories are integrated using uncertainties introduced into the system models but with the nominal optimal control law applied to the system. The second approach instead re-optimizes the control law, in the presence of uncertainties to determine the effect on the vehicle performance, in this case using the final vehicle mass into orbit as the figure of merit. A statistical analysis is conducted on the results in order to produce an expectancy curve which relates the probability of the vehicle having a certain performance to the expected variation due to uncertainty in the system design parameters.

The foundation of an ideal robust and reliable method for trajectory optimisation is an algorithm which guarantees convergence, with a high level of confidence, to the global optimum of the system. The convergence of traditional gradient-based optimal control solvers is severely compromised by the presence of discontinuities in any of the design models, and it can be difficult, if not impossible, to find a valid optimal solution using this approach if a good initial guess is not properly chosen. One of the interesting elements of the work presented here is that a hybrid stochastic-deterministic approach to the optimization of the system has been used in order to overcome the limitations of traditional gradient-based techniques.

The paper starts by describing the methods and tools that were used to solve the trajectory optimisation problem for the representative space plane and to characterize the various uncertainties in the models that were used in its design. In the subsequent section of the paper, the composition of the various models that comprise the overall system design for the space plane are described in more detail. The specific trajectory optimisation problem is then addressed, followed by a discussion of the results.

\section{Optimisation and robustness analysis}

The following section provides background into the different types of optimisation approaches that are used in this work to determine the optimal control law for the ascent trajectories of the space access vehicles and explains the rationale behind the approach that has been adopted.

\section{A. Trajectory optimisation}

All practical methods for solving optimal control problems involves an approach which discretizes the system into a finite set of unknowns. Generally, the dynamical system, characterized by a set of continuous functions, is transcribed into a problem with a finite set of variables, following which the resultant finite dimensional problem is solved by using a numerical parameter optimisation method. A further step is required to assess the accuracy of the finite dimensional approximation to the continuous problem. This methodology allows the optimal control problem to be re-written as a non-linear programming problem (NLP).

There are several methods that can be used to transcribe an optimal control problem into a NLP. ${ }^{4}$ The easiest approach is the direct shooting method, where only the system controls are discretized and the whole optimisation process iterates over the integration of dynamical system from initial to final conditions. The disadvantage of the approach is that the objective function and the constraints on the optimization can only be evaluated at the end of the simulation. Transcription by the single shooting approach results in a NLP problem with a relatively small number of independent design variables, but dynamical systems which are subject to instability for certain values of their control parameters (as is often the case) are difficult to treat with this method. A more sophisticated approach is the direct collocation method where both the controls and the state variables defining the system are discretized in terms of the elapsed time. In this way, an infinite-dimensional ODE system is replaced by a finite number of equality constraints, and the integrals associated with the objective and constraint functions of the original problem are approximated. This approach gives a large but sparse NLP, which can be solved conveniently using any one of a number of sequential quadratic programming (SQP) solvers. $^{5}$

The proposed trajectory optimization approach is based on a mixed formulation which combines a population-based stochastic algorithm with a deterministic gradient-based method. Population-based stochastic algorithms are able to explore the global search space very efficiently, and are thus able to find feasible solutions when the constraints on the system are sufficiently loose and the dimensionality of the problem is not high (generally less than 100 design variables). Sampling of the search space can become inefficient, 
however, if the constraints on the system are overly strict. On the other hand, deterministic gradient based solvers can deal efficiently with equality constraints and problems with many dimensions (generally many more than 100 design variables). The idea here is to use a stochastic approach coupled to a single shooting transcription method to find an initial solution, in terms of the variation of the system states and controls with time, for the optimized trajectory by first relaxing the constraints on the final states of the system. Then this solution is used as a starting point for a further local optimisation by the direct collocation method. This second step is intended to both improve the value of the objective function and to ensure that the equality constraints on the required final states are satisfied.

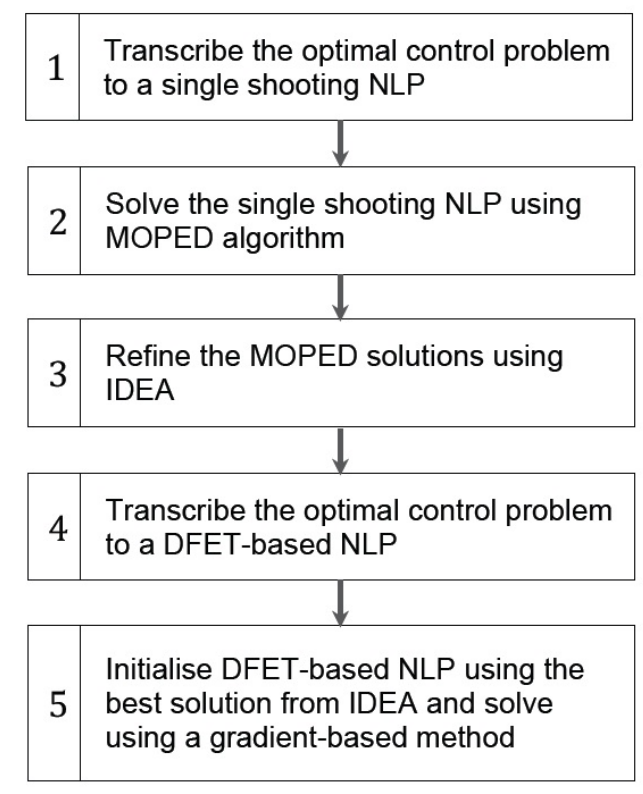

Figure 1: General scheme of the trajectory optimisation process.

A general overview of the optimisation process that was used in the work described in this paper is shown in Fig. 1 and is described in more detail as follows.

1. Transcribe the optimal control problem into a single shooting NLP As a first step, the optimal control problem is converted to a problem with a finite set of variables by using a direct single shooting approach and relaxing the constraints on the system.

2. Solve the single shooting NLP using MOPED The NLP is then solved using a hybrid Evolutionary Algorithm (EA) which is obtained by coupling together two different algorithms: the MultiObjective Parzen-based Estimation of Distribution ${ }^{6}$ (MOPED) and a modified version of the Inflationary Differential Evolution Algorithm ${ }^{7,8}$ (IDEA).

The MOPED algorithm belongs to a subset of Evolutionary Algorithms called Estimation of Distribution Algorithms ${ }^{9}$ (EDA). Within these algorithms the typical evolutionary search operators, such as crossover and mutation, are replaced with statistical tools. These tools build an approximate probabilistic model of the search space, with the role of the crossover and mutation operators replaced by appropriate sampling of this model. MOPED is an EDA that can be used for multi-objective optimisation of continuous problems, and uses the Parzen method ${ }^{10}$ to build a probabilistic representation of Pareto solutions and can handle multivariate dependencies of the variables. ${ }^{6,11}$ This EDA optimizer implements the general layout and selection techniques of the Non-dominated Sorting Genetic Algorithm II (NSGA-II), ${ }^{12}$ but with the traditional crossover and mutation search approaches of NSGA-II replaced by sampling of the Parzen model. NSGA-II was chosen as the basis for MOPED mainly due to its simplicity and for the quality of the results that are obtained using this approach for various diverse optimisation problems. The Parzen method uses a non-parametric approach to kernel density estimation. It allocates $N_{\text {ind }}$ identical elementary probability density functions (PDF) (where $N_{\text {ind }}$ is the number of individuals within the current population), each one centered on a different element of 
the sample. A probabilistic model of the promising search space portion is built on the basis of the statistical data provided by $N_{\text {ind }}$ individuals, and new individuals are sampled by the probabilistic model itself. The variance of each elementary PDF depends on $i$ ) the location of the individuals in the search space and $i i$ ) the fitness value of these individuals. The way the algorithm is constructed leads to values that favor sampling in the neighborhood of the most promising solutions. The features of MOPED, which is used here for a single objective problem, allows for an efficient exploration of the search space but often prevent fine convergence on the optimal point particularly when the solutions of the final population are spread over different areas which are far apart from each other in the feasible space. This feature of the approach has prompted the idea of coupling MOPED with another EA which has better convergence properties. To this end, IDEA has been selected.

3. Refine MOPED solution using IDEA The Inflationary Differential Evolution Algorithm (IDEA) is based on a hybridization of a differential evolution ${ }^{13}$ (DE) variant and the logic behind monotonic basin hopping ${ }^{14}(\mathrm{MBH})$. The resulting algorithm has been demonstrated to out-perform both DE and $\mathrm{MBH}$ on some difficult space trajectory design problems, for instance those where the search space has a (multi) funnel-like structure. ${ }^{7}$ The main features of the IDEA algorithm are reported in depth by Vasile et al. $;^{7}$ here just the more practical aspects of the currently adopted algorithm are discussed. In essence, the final solutions obtained by MOPED are clustered based on the Euclidean distance between them in the search space, resulting in a variable number of solution clusters. The distance threshold for each cluster is chosen such that each cluster should have a number of individuals in the range $\left[N_{c l, l}, N_{c l, u}\right]$. A DE process is then performed a number of times, beginning with the sub-population of each cluster. Each process is stopped only when the population contracts to below a predefined threshold. Every time the DE process stops, a local search is performed in order to properly converge to the local optimum. As the design optimisation in this case is constrained, the internal DE mechanism can be modified such that the comparison of individuals during the DE process is able to account for the constraints on the system. In the unconstrained DE algorithm, ${ }^{13}$ each parent solution is compared with its offspring; the solution with the best objective function value is chosen to be part of the population of the next generation. Instead, for the constrained DE algorithm used here, when parents and offspring are compared, the solutions are first evaluated in terms of constraint compatibility. Evaluating the solutions based first on how well they comply with the constraints ensures that each solution is valid before optimality in terms of the objective function for the system is assessed.

4. Transcribe the optimal control problem using DFET The optimal control problem is converted to a problem with a finite set of variables using a direct collocation method based on Finite Elements in Time (DFET) on a spectral basis. ${ }^{15}$

5. Initialize DFET-based NLP using best solution from IDEA and solve using gradient method The NLP problem is then solved by a gradient-based optimisation method, which uses the solution obtained from the previous stochastic optimisation as a starting point.

\section{B. Robust design and uncertainties}

Within the early stages of design, where models and simulations are used to assess preliminary design options, it is important to characterize the uncertainty affecting the models and to consider their impact on the design in order to properly assess the performance of the system. Uncertainties occur across all of the different phases of modeling and simulation, and can be categorized either as aleatory uncertainties, which are due to the inherent random behavior of a system, or epistemic uncertainties that arise from a lack of knowledge about a system.

Once the levels of uncertainty within the system have been estimated and implemented, they can be used to $i$ ) estimate the sensitivity of the control law(s) to the uncertainties themselves (robustness analysis), $i i$ ) obtain a statistical characterization of the likely vehicle performance in the presence of uncertainty, and/or iii) obtain a robust optimization of the trajectory, in other words to optimize the performance of the system while minimizing the effect of uncertainties. In this paper the estimated uncertainties have been used to analyze the robustness of the nominal control law for the vehicle and to obtain a statistical characterization of the vehicle performance in the presence of uncertainty in some of its key design variables.

Two types of uncertainty-based analyses are performed. The first test is aimed at evaluating the robustness of the nominal control law against the estimated uncertainties. Once the optimisation has been 
performed using nominal models, the nominal control law is reintegrated using atmospheric and aerodynamic models whose output variables have been perturbed relative to their nominal values. The second test instead is aimed at evaluating the optimal performance of the vehicle subject to uncertainties within the optimisation loop. Multiple optimizations are performed, each one with a different randomized set of perturbations in the atmospheric and aerodynamic models.

The level of uncertainty on a specific variable is represented by a percent deviation around the nominal value. Specifically, the atmospheric temperature and pressure are perturbed as any uncertainty considered for these two quantities affects other atmospheric values, such as the density and speed of sound, as well as other models such as the aerodynamics and propulsion which lead to altering the trajectory dynamics and ultimately the control. Uncertainties within the aerodynamics model specifically are evaluated by perturbing the net lift and drag forces.

Once a deterministic solution is available from the atmospheric and aerodynamic models, uncertainties are introduced by perturbing the nominal values of the outputs of the models. The value of a generic uncertain quantity, $x_{n o m}$, in the presence of uncertainty, $x_{u n c}$, can be defined as:

$$
x_{u n c}=x_{n o m}+\varepsilon S_{E} x_{n o m}
$$

where $\varepsilon$ is the uncertainty bounding function which depends on the operational conditions, and $S_{E}$ is a sampling surface mapping the set of operating conditions into the interval $[-1,1]$. The function $S_{E}$ is defined as an interpolating surface dependant on the value of each interpolating node. Each time a new perturbation profile is needed, a new set of values for the parameters defining $S_{E}$ is randomly generated. For the robustness analysis of the nominal control law, the process generates $n$ different $S_{E}$ surfaces by randomly choosing the values defining $S_{E}$ from an uniform distribution in the interval [-1,1], where $n$ is the total number of integrations performed. For the multiple optimisation robustness analysis, the same set of $n S_{E}$ surfaces are used to generate $n$ optimised trajectories accounting for uncertainty.

\section{System Models}

The following section presents the mathematical models used to simulate the vehicle performance, specifically the propulsion system, vehicle aerodynamics, atmospheric model and the trajectory dynamics. As the vehicle configuration is based on a horizontal take-off, the full ascent profile should include a runway take-off followed by a subsonic climb-out. For this analysis however the complexities of the near-ground segment were ignored. The ascent profile instead starts just after the transition into the supersonic regime, at an altitude of around $8 \mathrm{~km}$.

\section{A. Dynamic model}

The vehicle is considered to be a point with variable mass flying around a spherical, rotating earth. The dynamics of the vehicle along the trajectory is governed by the following set of differential equations: ${ }^{16}$

$$
\begin{aligned}
\dot{h}= & v \sin \gamma \\
\dot{v}= & \frac{F_{T} \cos \epsilon-D}{m}-g \sin \gamma+\omega_{e}^{2}\left(R_{\mathrm{E}}+h\right) \cos \lambda(\sin \gamma \cos \lambda-\cos \gamma \sin \chi \sin \lambda) \\
\dot{\gamma}= & \frac{F_{T} \sin \epsilon+L}{m v} \cos \mu-\left(\frac{g}{v}-\frac{v}{R_{\mathrm{E}}+h}\right) \cos \gamma+2 \omega_{\mathrm{E}} \cos \chi \cos \lambda \\
& \quad+\omega_{\mathrm{E}}^{2}\left(\frac{R_{\mathrm{E}}+h}{v}\right) \cos \lambda(\sin \chi \sin \gamma \sin \lambda+\cos \gamma \cos \lambda) \\
\dot{\chi}= & \frac{L}{m v \cos \gamma} \sin \mu-\left(\frac{v}{R_{\mathrm{E}}+h}\right) \cos \gamma \cos \chi \tan \lambda \\
& \quad+2 \omega_{\mathrm{E}}(\sin \chi \cos \lambda \tan \gamma-\sin \lambda)-\omega_{\mathrm{E}}^{2}\left(\frac{R_{\mathrm{E}}+h}{v \cos \gamma}\right) \cos \lambda \sin \gamma \cos \chi \\
\dot{\lambda}= & \frac{v \cos \gamma \sin \chi}{R_{t}+h} \\
\dot{\theta}= & \frac{v \cos \gamma \cos \chi}{\left(R_{t}+h\right) \cos \lambda}
\end{aligned}
$$


where $h$ is the altitude above mean sea level, $v$ is the absolute velocity in a rotating Earth-centered reference frame, $\gamma$ is the flight path angle, $\chi$ is the path direction angle, $\mu$ is the bank angle, $\lambda$ is the latitude, $\theta$ is the longitude, $m$ is the mass of the vehicle, $F_{T}$ is the magnitude of the thrust given by the engine, $L$ and $D$ are the aerodynamic lift and drag forces, respectively, $R_{\mathrm{E}}=6375 \mathrm{~km}$ is the mean Earth radius, $\omega_{\mathrm{E}}=7.2921 \times 10^{-5} \mathrm{rad} / \mathrm{s}$ is the rotational velocity of the Earth, $g_{0}=9.80665 \mathrm{~m} / \mathrm{s}^{2}$ is the acceleration due to gravity at sea level and lastly $\epsilon=0.08727+\alpha$ rad is the angle between the thrust vector and the velocity vector of the vehicle, accounting for a $5^{\circ}$ thrust vector offset.

For simplicity, the control law only governs the angle of attack and thrust level, so no out of plane motion is considered $(\chi, \mu=0)$. In addition, the launch, ascent and orbit are all assumed to be contained within the equatorial plane $(\lambda=0)$.

\section{B. Earth model}

The gravitational field is assumed to be a function of the altitude and varies according to an inverse square law $g(h)=g_{0}\left(h /\left(R_{\mathrm{E}}+h\right)\right)^{2}$. The atmospheric characteristics (temperature, pressure, density and speed of sound) follow the US Standard Atmosphere 1976 model up to $1000 \mathrm{~km}$.

\section{Propulsion model}

As the vehicle is assumed to be propelled by a hybrid engine, two different models, one for the engine's air-breathing mode and another for its rocket mode, were developed. For both modes, the models give the maximum available thrust $F_{T}$ and propellant consumption $\dot{m}_{P}$ as a function of altitude $h$, Mach number $M$ and atmospheric conditions.

The numerical model for the air-breathing propulsion system is based on a modified analysis of a ramjettype engine. The stagnation temperature $T_{0}$ at the intake of the engine is a function of the flight Mach number and is assumed to be that which results from isentropic compression of the oncoming flow. Losses in the intake are manifest as a reduction in stagnation pressure at the exit of the intake diffuser compared to the ideal case, as specified using a simple pressure recovery factor. For present purposes, a constant efficiency is assumed for the intake, and this results in a pressure recovery factor between 0.75 and 0.95 , depending on the flight Mach number.

As the flight Mach number increases, the temperature rise due to compression in the intake increases rapidly. If this temperature rise becomes too high then this limits the amount of energy that can be added during the combustion process and thus the thrust that can be produced by the engine. This effect is mitigated in the present analysis by setting the post-combustion temperature based on the flight Mach number and altitude in order to ensure that sufficient energy can be input into the combustion chamber and thus an appropriate level of thrust can be maintained.

Fuel usage in the combustion chamber is determined from an energy balance across the combustion chamber, with the actual fuel-air ratio, $f_{a}$, given by,

$$
f_{a}=\frac{c_{P g} T_{04}-c_{P} T_{03}}{\eta_{c o m b}\left(Q_{r}-c_{P g} T_{04}\right)}
$$

where $T_{03}$ and $T_{04}$ are the temperatures at the start and end of combustion respectively, $c_{P}$ and $c_{P g}$ are the specific heats of the gas before and after combustion respectively, $Q_{r}$ is the heating value of the fuel, here assumed to be hydrogen, and $\eta_{c o m b}$ is the combustion efficiency. A pressure drop across the combustion chamber of $4 \%$ is assumed.

The exit nozzle of the engine is assumed to expand the flow to ambient pressure, and the resulting nozzle exit static temperature is used to determine the exit Mach number and hence jet velocity. Expansion through the nozzle is assumed to have a constant adiabatic efficiency of $93 \%$.

The overall mass flow for the engine, $\dot{m}_{e n g}$, is given by multiplying the nozzle exit area by the exit velocity and the static density. The fuel mass flow $\dot{m}_{P i}$ for the engine is then obtained by multiplying the fuel-air ratio, $f_{a}$ in Eq. (3), by the engine mass flow. The total fuel usage $\dot{m}_{P, \text { ramjet }}$ of the vehicle is determined by scaling $\dot{m}_{P i}$ by the number of engines on the airframe, assuming all engines are operating at identical conditions.

The total thrust for a single engine is given by the general thrust equation

$$
F_{T, \text { ramjet }}=\dot{m}_{e n g}\left(\left(1+f_{a}\right) v_{e}-v_{f l}\right)
$$


where $v_{e}$ is the nozzle exit velocity, and $v_{f l}$ is the flight velocity, determined from the flight Mach number and atmospheric temperature. The total thrust delivered to the airframe is determined by scaling $F_{T}$ by the number of engines and again assuming that all engines are operating at the same conditions.

Figure 2 shows the overall thrust and fuel consumption as a function of altitude and Mach number for the air-breathing engine model.

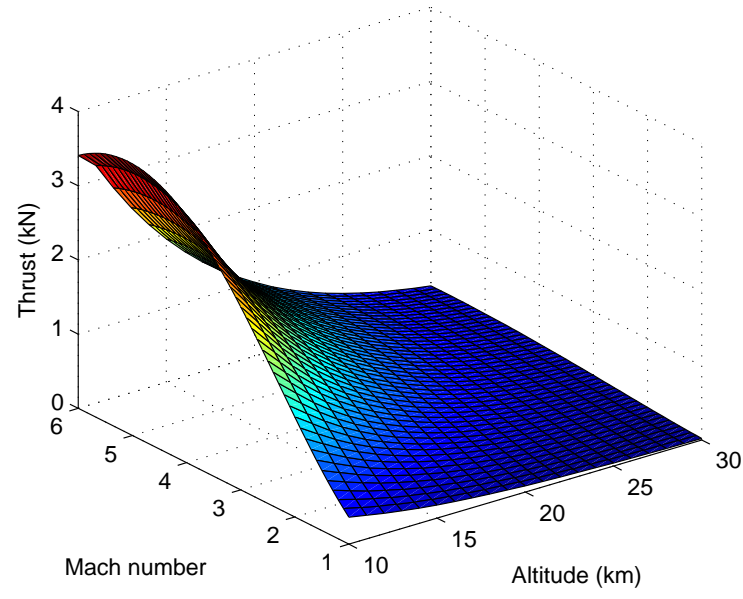

(a) Thrust $F_{T, \text { ramjet }}$ versus Mach number $M$ and altitude $h$

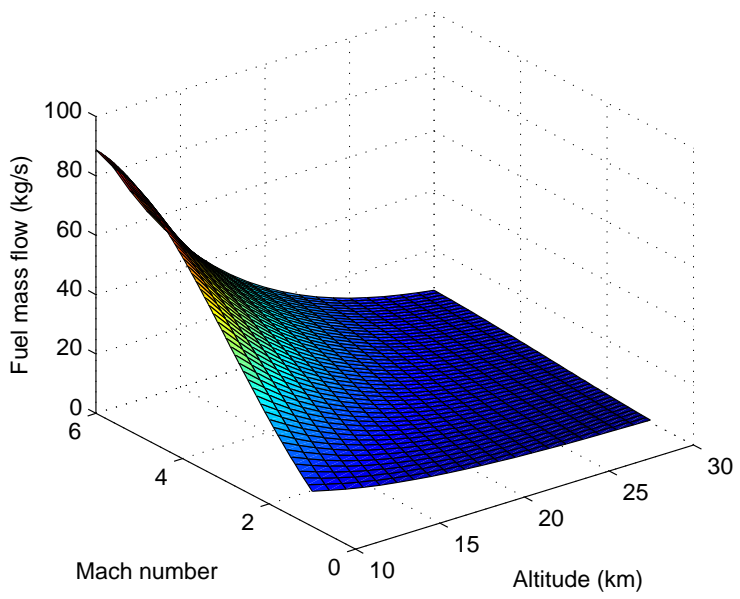

(b) Fuel consumption $\dot{m}_{P, \text { ramjet }}$ versus Mach number $M$ and altitude $h$

Figure 2: Propulsion model outputs for the air-breathing ramjet-type engine.

Once the vehicle nears hypersonic speeds, the engine transitions from air-breathing mode to rocket mode. When the engine is in rocket mode, the basic rocket equation is used to determine the thrust level $F_{T}$, and the specific impulse $I_{s p}$ is then used to determine the propellant mass flow according to,

$$
\begin{aligned}
F_{T, \text { rocket }} & =\dot{m}_{P} v_{e}+\Delta P A_{e} \\
\dot{m}_{P, \text { rocket }} & =\frac{F_{\text {rocket }}}{g_{0} I_{s p}}
\end{aligned}
$$

where $A_{e}$ is nozzle area at the exit, and $\Delta P$ is the difference between the free stream and exit pressures. A specific impulse of $460 \mathrm{~s}$ was assumed.

Finally, a throttle setting is applied as part of the control law such that both the applied thrust and mass flow are scaled proportionally,

$$
\begin{aligned}
F_{\text {applied }} & =\delta_{T} F_{T} \\
\dot{m}_{P, \text { applied }} & =\delta_{T} \dot{m}_{P}
\end{aligned}
$$

\section{Aerodynamic model}

A simple algebraic model is used to calculate the aerodynamic forces acting on the vehicle as a function of angle of attack $\alpha$ and flight Mach number $M$. At low supersonic Mach numbers, the lift on the vehicle is modeled as that due to a delta wing at a given angle of attack, as per linearized aerodynamic theory so that,

$$
C_{L, s s}=\frac{C_{L \alpha}}{\sqrt{M^{2}-1}} \frac{A_{\text {wing }}}{A_{\text {ref }}} \sin \alpha \cos \alpha
$$

where $A_{\text {wing }}$ is the area of the lifting surfaces of the vehicle and $C_{L \alpha}$ is a constant that is dependent on the geometry of the vehicle. As the flight Mach number is increased towards the hypersonic regime, the nonlinear variation of lift coefficient with angle of attack becomes progressively more important. An additional contribution to the lift coefficient,

$$
C_{L, h s}=2 \frac{A_{w i n g}}{A_{\text {ref }}} \sin ^{2} \alpha \cos \alpha
$$


is thus calculated according to Newtonian flow theory ${ }^{17}$ and the variation with Mach number of the overall lift coefficient that is generated by the vehicle is then modeled as:

$$
C_{L}=\frac{C_{L, s s}+C_{L, h s}}{2}+\sqrt{\left(C_{L, s s}\right)^{2}+\left(C_{L, h s}\right)^{2}}
$$

The drag coefficient of the vehicle is calculated simply as:

$$
C_{D}=C_{D_{0}}(M)+C_{L} \tan \alpha
$$

where the first term $C_{D_{0}}$, a function of the Mach number, accounts for the wave, base, and viscous drag of the vehicle and the second term is the induced drag of its lifting surfaces. The overall lift and drag forces on the vehicle are then obtained by multiplying by the dynamic pressure $q$ and the vehicle reference area $A_{\text {ref }}$.

\section{Test Case}

Due to the nature of the hybrid engine, the ascent trajectory is modeled as two phases: a first phase with the engine in air-breathing mode, and a second phase with the engine in rocket mode.

For the trajectory optimisation problem, the control vector $\mathbf{c}=\left[\boldsymbol{\alpha}, \boldsymbol{\delta}_{T}, t_{1}, t_{2}\right]$, where $\boldsymbol{\alpha}(t), \boldsymbol{\delta}_{T}(t)$ give the time schedule of the angle of attack and throttle setting respectively, and $t_{1}, t_{2}$ are the durations of the first and second phase with the engine mode switching from air-breathing to rocket propulsion at $t_{1}$. The search space $D$ for the controls is defined by the following bounds: $\alpha \in[-1,1] \mathrm{rad}, \delta_{T} \in[0,1]$ (i.e., from 0 to $100 \%$ of the maximum available thrust), and the flight time for each phase $t_{1}, t_{2}$ cannot exceed $1800 \mathrm{~s}$, where the total flight time is $t_{f}=t_{1}+t_{2}$. The control law is the result of the optimal control problem maximizing the final vehicle mass,

$$
\max _{\mathbf{c} \in D}\left(m\left(t=t_{f}\right)\right)
$$

subject to dynamics in Eq. (2) plus initial conditions set to start after the transition into the supersonic regime:

$$
\begin{aligned}
& h(t=0)=h_{0}=8.2 \mathrm{~km} \\
& v(t=0)=v_{0}=0.470 \mathrm{~km} / \mathrm{s} \\
& \gamma(t=0)=\gamma_{0}=8 \mathrm{deg} \\
& \chi(t=0)=\chi_{0}=0 \mathrm{deg} \\
& \lambda(t=0)=\lambda_{0}=0 \mathrm{deg} \\
& \theta(t=0)=\theta_{0}=0 \mathrm{deg} \\
& m(t=0)=m_{0}=261380 \mathrm{~kg}
\end{aligned}
$$

The terminal conditions: $h\left(t=t_{f}\right)=h_{f}=80 \mathrm{~km}, v\left(t=t_{f}\right)=v_{f}=7.380 \mathrm{~km} / \mathrm{s}$ and $\gamma\left(t=t_{f}\right)=\gamma_{f}=$ $0 \mathrm{deg}$ are those required to enter into a circular $80 \mathrm{~km}$ altitude orbit, in a rotating Earth reference frame. Link constraints are enforced between the two phases to guarantee the required continuity on both the states and controls.

$$
\begin{aligned}
& h_{f, 1}=h_{0,2} \\
& v_{f, 1}=v_{0,2} \\
& \gamma_{f, 1}=\gamma_{0,2} \\
& m_{f, 1}=m_{0,2} \\
& \alpha_{f, 1}=\alpha_{0,2}
\end{aligned}
$$

Additional path constraints based on operating conditions are imposed for both phases on the maximum axial acceleration $a_{x}(t) \leq 30 \mathrm{~m} / \mathrm{s}^{2}$, maximum normal acceleration $a_{z}(t) \leq 30 \mathrm{~m} / \mathrm{s}^{2}$, dynamic pressure through the equivalent air speed $E A S(t) \leq 335 \mathrm{~m} / \mathrm{s}$, and for the maximum Mach number in the air-breathing mode $M\left(t \leq t_{1}\right) \leq 5.4$.

\section{Shooting based evolutionary optimisation}

For the evolutionary optimisation, the control vector $\mathbf{c}$ is composed of discrete values for the control law. For each solution, the control law is interpolated using a piecewise cubic interpolation and directly integrated 
in time. The NLP problem to solve in this case is relatively small and can be handled by an evolutionary approach.

Within the control vector $\mathbf{c}$, the angle of attack is discretized into 9 variables or elements for the first air-breathing phase, $\mathbf{c}(1: 9) \in[0,30]$ deg and 8 elements for the second rocket phase, $\mathbf{c}(10: 17) \in[-5,30]$ deg. The throttle control setting for the first phase is preset to $\delta_{T}=1$, while the second phase is discretized into 9 elements $\mathbf{c}(18: 26) \in[0.5,1]$. The last 2 design variables define the duration of first and second phase respectively, $\mathbf{c}(27,28) \in[80,1800]$ s. The equality constraints on final states are converted into inequality constraints such that $h_{f} \in[80,82] \mathrm{km}, v_{f} \in[7.34,7.40] \mathrm{km} / \mathrm{s}$, and $\gamma_{f} \in[-2,2] \mathrm{deg}$.

\section{Finite element in time direct collocation}

When solved by FET direct collocation method, the trajectory is decomposed into $N$ elements, each of which has $n_{c}$ collocation points. After transcription, the optimal control problem including the initial and final conditions given above becomes the general NLP problem with the objective function,

$$
\max _{\boldsymbol{\alpha}_{s}, \boldsymbol{\delta}_{s}, \mathbf{t}_{s}}\left(m\left(t=t_{f}\right)\right)
$$

subject to the nonlinear algebraic constraints in the form,

$$
C\left(\mathbf{h}_{s}, \mathbf{v}_{s}, \boldsymbol{\chi}_{s}, \boldsymbol{\lambda}_{s}, \boldsymbol{\theta}_{s}, \mathbf{m}_{s}, \boldsymbol{\alpha}_{s}, \boldsymbol{\delta}_{s}, \mathbf{t}_{s}\right)=0
$$

where $\mathbf{h}_{s}, \mathbf{v}_{s}, \boldsymbol{\chi}_{s}, \boldsymbol{\lambda}_{s}, \boldsymbol{\theta}_{s}, \mathbf{m}_{s}, \boldsymbol{\alpha}_{s}, \boldsymbol{\delta}_{s}, \mathbf{t}_{s}$ are vectors containing the set of discrete points for each of the state, control, and time variables at each node defined by the transcription scheme. Due to its high dimensionality of the NLP problem, a deterministic large-scale method was used over a more conventional stochastic approach, such DE.

\section{A. Implementation of uncertainty}

For the two atmospheric parameters, temperature and pressure, the uncertainty distribution function in Eq. (1) is given by,

$$
\varepsilon_{a t m}(h)=l_{b, a t m}\left(1-\frac{h}{h_{c}}\right)+u_{b, a t m}\left(\frac{h}{h_{c}}\right)
$$

where $l_{b, a t m}$ and $u_{b, a t m}$ are, respectively, the lower and upper boundaries defined as the percentage around the nominal value, and $h \in\left[0, h_{c}\right]$ where $h_{c}=150 \mathrm{~km}$ is the cut-off value for the increase, here set equal to the maximum value of the altitude vector. For the atmospheric temperature, the boundaries of the perturbations are between $l_{b, T}=10 \%$ and $u_{b, T}=50 \%$, while for pressure the bounds are set to between $l_{b, P}=1 \%$ and $u_{b, P}=50 \%$. Figure 3 shows an example of the approach applied to the temperature showing the nominal profile compared to a perturbed profile due to uncertainties.

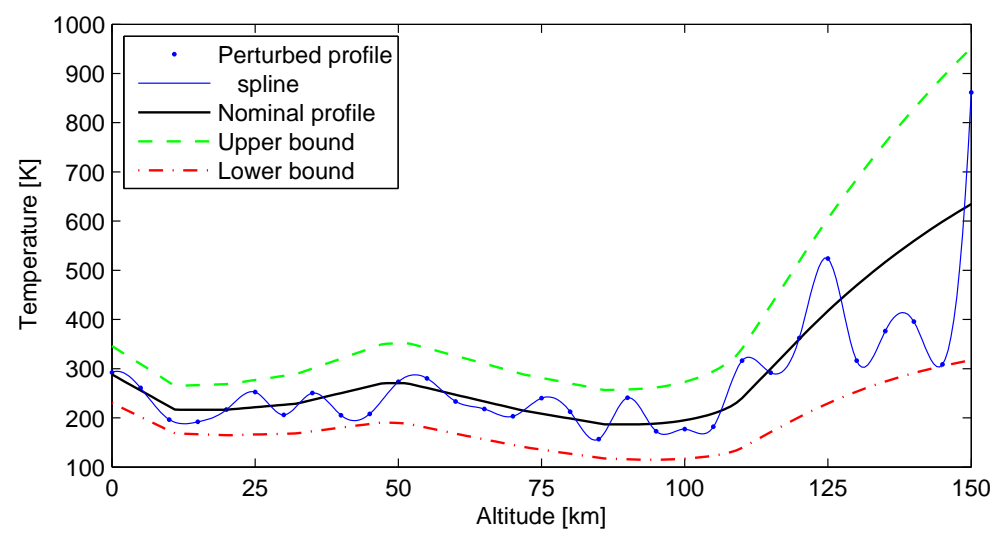

Figure 3: Nominal and perturbed values for the atmospheric temperature as a function of altitude.

In general, the level of uncertainty in atmospheric modeling increases with altitude. Specifically with the US 1976 Standard Atmosphere model used here, below $32 \mathrm{~km}$ the model is well known and identical 
to the Standard Atmosphere of the International Civil Aviation Organization (ICAO), however above that, and more notably above the ozone layer at $86 \mathrm{~km}$ the model becomes less accurate when compared with other complex models based on more recent experimental data. ${ }^{18}$ Other key contributors to uncertainty are the stochastic nature of the radiation pattern from the Sun on the Earth's surface and differences between geographic locations. A certain level of error is unavoidable when creating an averaged, globally applicable atmospheric model.

Aleatory uncertainties in the aerodynamic model arise, for instance, from the unknown effects of turbulence and any unsteadiness in the flow over the vehicle, while epistemic uncertainties, particularly in the high altitude or high speed regimes, arise from basic deficiencies in understanding the role of flow rarefaction and gas chemistry in governing the loads generated on the vehicle.

The uncertainty distribution function for the aerodynamic model is a function of three parameters within the model: the altitude, Mach number and angle of attack although is applied only to the lift and drag force variables. The bounds of the uncertainties are assumed to increase linearly with altitude, Mach number and the absolute value of the angle of attack.

$$
\begin{aligned}
\varepsilon_{\text {aero }}(h, M, \alpha)= & \left(l_{b, h}\left(1-\frac{h}{h_{c}}\right)+u_{b, h}\left(\frac{h}{h_{c}}\right)\right)+\left(l_{b, M}\left(1-\frac{M}{M_{c}}\right)+u_{b, M}\left(\frac{M}{M_{c}}\right)\right) \\
& +\left(l_{b, \alpha}\left(1-\frac{\alpha}{\alpha_{c}}\right)+u_{b, \alpha}\left(\frac{\alpha}{\alpha_{c}}\right)\right)
\end{aligned}
$$

where $h \in\left[0, h_{c}=150\right] \mathrm{km},|\alpha| \in\left[0, \alpha_{c}=1\right] \mathrm{rad}, M \in\left[0, M_{c}=40\right]$. The lower and upper bounds are set to $10 \%$ and $20 \%$ respectively, and are the same for all three parameters. As the uncertainty function is dependant on multiple sources it means that the perturbations can be as much as $\pm 30 \%$ of the nominal value when the vehicle is at Mach 0 at sea level with a $0^{\circ}$ angle of attack, and can be as much as $\pm 60 \%$ of the nominal value if the vehicle flies at Mach 40 at an altitude of $150 \mathrm{~km}$ with a $57.3^{\circ}$ (1 rad) angle of attack.

The uncertainty distribution function $\varepsilon_{a e r o}$ is designed to reflect the expected margins of uncertainty of the aerodynamic model used here, which increases with the altitude and velocity since the model cannot accurately predict the characteristics of the vehicle when flying at very high speed through the rarefied flow regime. The increase of the uncertainty bounds with the increase in angle of attack represents the inability to properly model the complex phenomena that occur at high angles of incidence, such as flow separation.

These uncertainty models, in particular the boundary conditions and uncertainty distribution functions, are general approximations and intentionally overestimated the potential uncertainties. Future work will be aimed at properly characterizing the uncertainties within the system by appropriate physical modelling.

\section{Simulation Results}

Following the procedures described above, a baseline trajectory was optimized using the nominal values from the models, i.e., not affected by any uncertainty. Figure 4 shows the optimized control law for the angle of attack $\alpha$ (see Fig. 4a) and the throttle setting $\delta_{T}$ (see Fig. 4b) as a function of time. The figures also show the timings and transition point where the engine switches from air-breathing to rocket propulsion.

In order to analyze the sensitivity of the nominal control law to uncertainties in the system models, the set of controls shown in Fig. 4 were integrated 100 times with different randomized atmosphere and aerodynamic model parameters. The evolutions of state vectors with time are showed in Fig. 5. As can be clearly seen none of the perturbed trajectories meet the final target conditions $(h=80 \mathrm{~km}, v=7.38$ $\mathrm{km} / \mathrm{s}, \gamma=0^{\circ}$ ). Figure 6 and Table 1 show the statistics on the deviation of the perturbed trajectories from the target conditions. In total, 94 of the 100 integrations converge towards the desired target conditions following a quasi-Gaussian distribution, while the remaining 6 computations diverge. Table 1 reports the mean value and standard deviation of the final states of the system both when all runs are taken into account ('full') and when diverging runs are discarded from the set ('clean').

Similarly, Fig. 7 shows the constraints on the EAS and axial acceleration throughout the trajectory. Compared to the nominal trajectory, which meets exactly the limit of the constraint in both cases, the majority of the perturbed trajectories violate the maximum allowable EAS and $a_{x}$ values. Figure 8 and Table 2 show the statistics of the two constraint variables. In the case of the EAS, even with the outlying values removed (so the clean set considering only 94/100 of the solutions), the mean EAS value is $431 \mathrm{~m} / \mathrm{s}$ compared to the constraint limit of $335 \mathrm{~m} / \mathrm{s}$. The axial acceleration is slightly closer to the maximum limit 


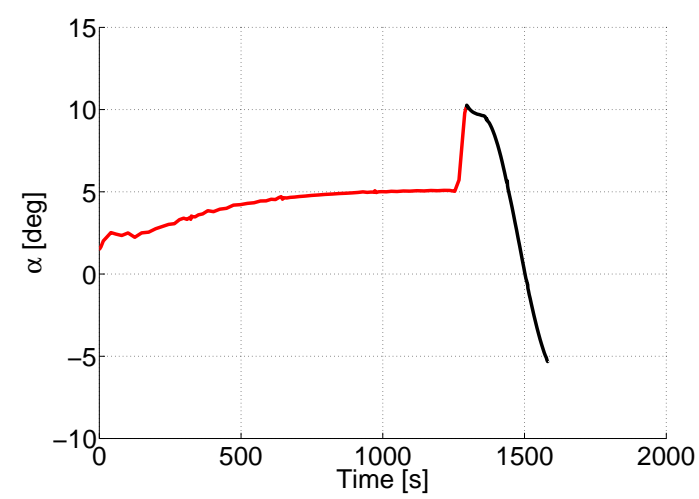

(a) Angle of attack

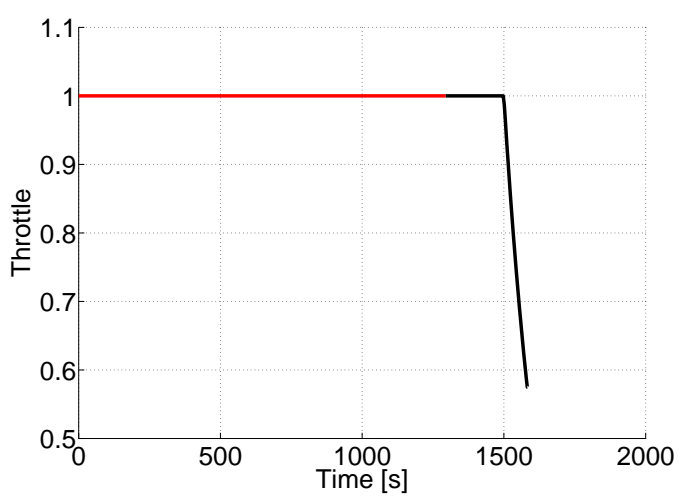

(b) Throttle setting

Figure 4: Nominal control laws where the red lines refer to primary air-breathing phase, while the black lines refer to the secondary rocket phase.

of $30 \mathrm{~m} / \mathrm{s}^{2}$, with the full and clean sets having similar mean values of $30.16 \mathrm{~m} / \mathrm{s}^{2}$ and $30.3 \mathrm{~m} / \mathrm{s}^{2}$, respectively, with small standard deviations of 0.86 and 0.67 .

Table 1: Statistics on the values of design parameters

\begin{tabular}{ccccc} 
State & $\begin{array}{c}\text { Mean } \\
(\text { full })\end{array}$ & $\begin{array}{c}\text { Standard deviation } \\
(\text { full })\end{array}$ & $\begin{array}{c}\text { Mean } \\
(\text { clean })\end{array}$ & $\begin{array}{c}\text { Standard deviation } \\
\text { (clean) }\end{array}$ \\
\hline Altitude, $h(\mathrm{~m})$ & 91649 & 51091 & 86158 & 29103 \\
Velocity, $v(\mathrm{~m} / \mathrm{s})$ & 7114 & 1028 & 7370 & 250 \\
Flight path angle, $\gamma$ deg & 6 & 29 & -0.1 & 2.7 \\
Final mass $m, \mathrm{~kg}$ & 56846 & 1941 & 56465 & 1293
\end{tabular}

Table 2: Statistics of path constraints.

\begin{tabular}{ccccc} 
Constraint & $\begin{array}{c}\text { Mean } \\
\text { (full) }\end{array}$ & $\begin{array}{c}\text { Standard deviation } \\
\text { (full) }\end{array}$ & $\begin{array}{c}\text { Mean } \\
\text { (clean) }\end{array}$ & $\begin{array}{c}\text { Standard deviation } \\
\text { (clean) }\end{array}$ \\
\hline Maximum $E A S(\mathrm{~m} / \mathrm{s})$ & 424 & 56.8 & 431 & 53 \\
Maximum $a_{x}\left(\mathrm{~m} / \mathrm{s}^{2}\right)$ & 30.16 & 0.86 & 30.3 & 0.67
\end{tabular}

Since the perturbed trajectories do not fully satisfy the various path and final constraints, the obtained values for the final vehicle mass cannot be used as a basis for comparison. As such, a second approach was used which performed 100 separate optimizations, each considering a different set of randomly perturbed atmospheric and aerodynamic parameters. The resultant control laws are shown in Fig. 9. The control laws all follow the same pattern or shape as the nominal one, and are able to exactly meet the desired final conditions (see Fig. 10) while also fulfilling all the path constraints. Figure 11 shows the values of the EAS and axial acceleration for the entire trajectory, demonstrating that the two constraints are satisfied over the whole trajectory.

The final vehicle masses that were obtained from the set of 100 optimizations of the control law can be statistically analysed. The mean values of the final mass are quite similar (56752 $\mathrm{kg}$ for the multiple optimisation case, and $56846 \mathrm{~kg}$ for the randomized integration of the nominal control law), while the standard deviations are quite different, with $702 \mathrm{~kg}$ for the multiple optimisation case and $1941 \mathrm{~kg}$ for the perturbed integration of the nominal control laws. 


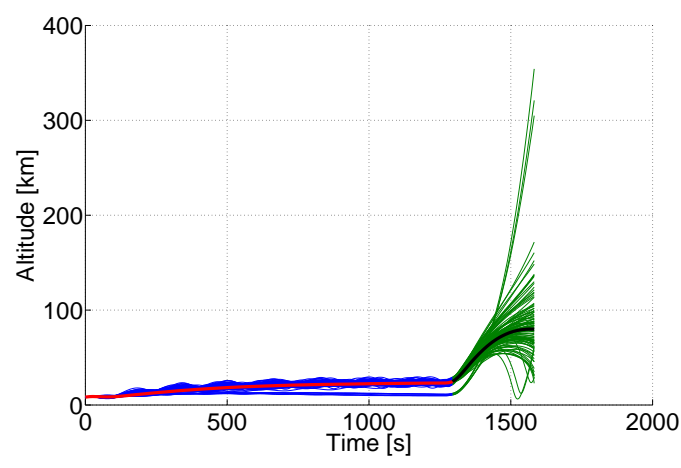

(a) Altitude

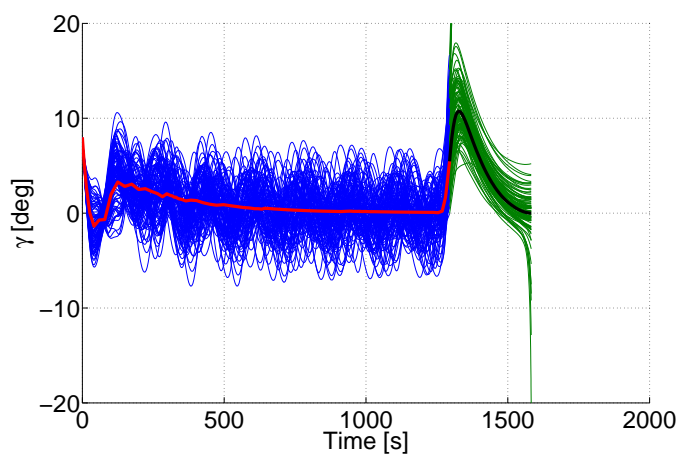

(c) Path angle

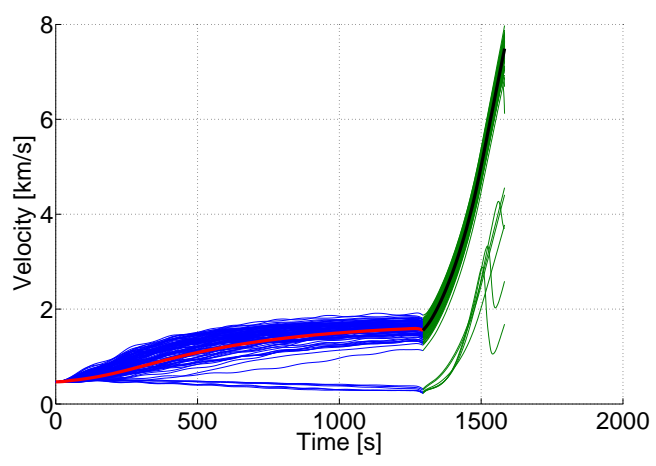

(b) Velocity

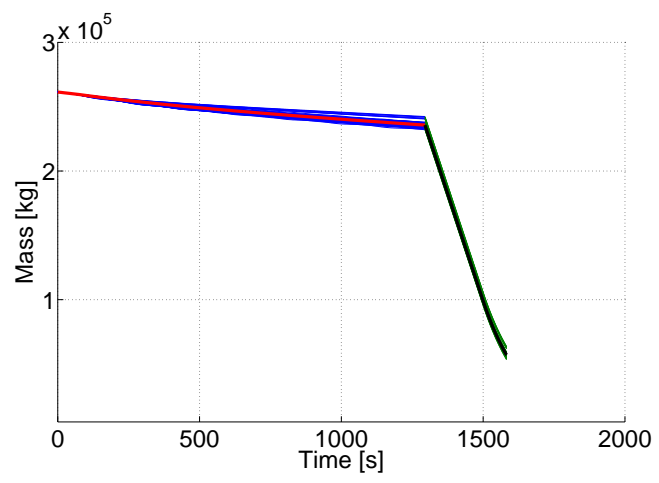

(d) Mass

Figure 5: Evolution of the states, where the bolded red and black lines refer to air-breathing and rocket phases respectively of the nominal trajectory, and the blue and green lines refer to air-breathing and rocket phases integrated with perturbations.

Figure 13 shows two cumulative distribution functions (CDF) representing the integration of the discrete PDFs given in Fig. 12 and Fig. 6d. The information summarized in Fig. 13 can give an engineer, during the preliminary design phase, an immediate understanding of the effect of uncertainties on the performance of the vehicle, shown here in terms of the final vehicle mass into orbit. The lower left side of the curve corresponds to the worst case scenario, when the effect of the uncertainty has the largest negative impact on the design which results in a very low final mass (in this analysis, every trajectory started with the same initial mass, thus the vehicle with largest final mass corresponds to the one with the lowest fuel consumption). On the other hand, the upper right side of the solid curve corresponds to the best case scenario, giving the highest maximum final mass that can be obtained given the effects of the uncertainty.

As it is a maximisation problem that is being considered, the CDF curves give the complement of the expectancy for obtaining a particular value of final mass between the best and worst case values. That is, if the uncertainties are correctly estimated, it can be guaranteed that in all cases (i.e., with a probability $p=1$ ) that the vehicle will arrive in orbit with a final mass which is equal to the value obtained in the worst case scenario (54768 $\mathrm{kg}$ in the case of the solid green curve), while there is a probability $p \leq 0.01$ that the vehicle will arrive into orbit with a mass greater or equal to the value obtained in the best case scenario (58400 $\mathrm{kg}$ in this case). It is therefore possible to have a direct quantification of the probability of a given final mass value between the best case and the worst case values, e.g., a final mass of $56188 \mathrm{~kg}$ can be obtained with $p=0.8$, while a final mass of $57279 \mathrm{~kg}$ can be obtained only with $p=0.2$.

It should be noted that while a sample size of only 100 is generally not considered large enough to draw accurate conclusions from the statistics, the sample size is large enough to test the validity and usefulness of this approach and to assess the potential for future work. 


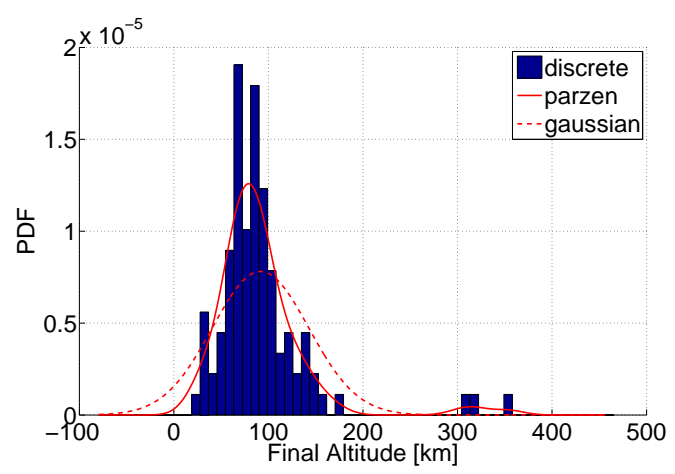

(a) Altitude

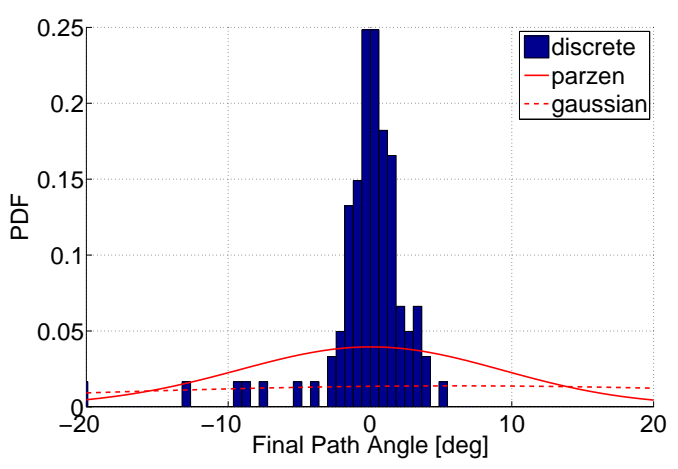

(c) Path angle

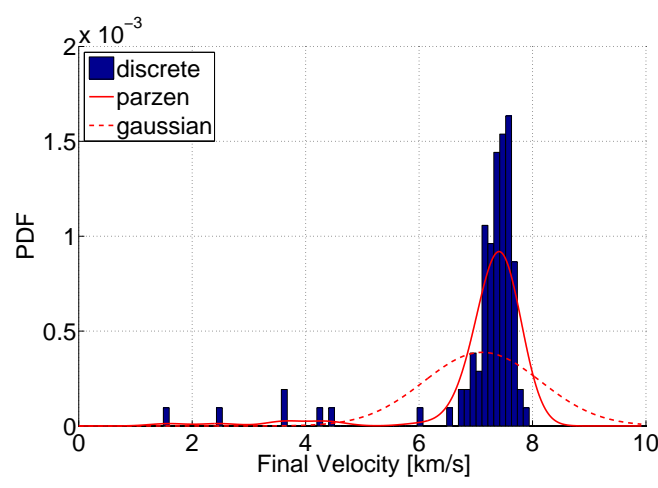

(b) Velocity

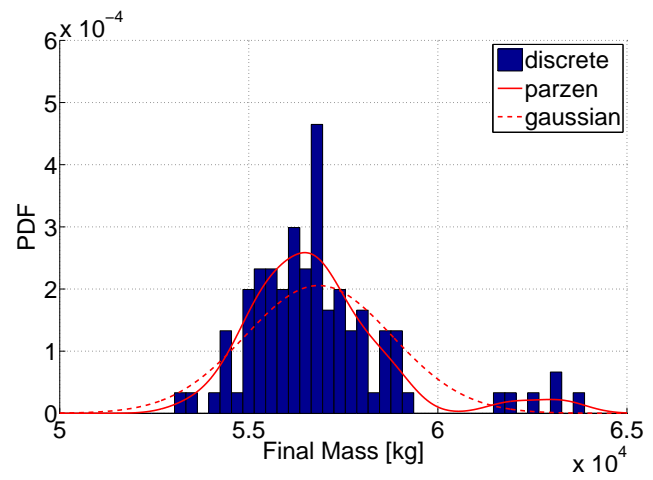

(d) Mass

Figure 6: PDF of the final states when the nominal control laws are integrated by means of perturbed atmospheric and aerodynamic models.

\section{Conclusion}

The paper describes a general approach for a trans-atmospheric trajectory optimisation. A hybrid stochastic-deterministic algorithm and its application for the design of ascent trajectories for a hybrid-engine, high performance, unmanned, single-stage-to-orbit vehicle for payload deployment into low Earth orbit have been detailed. Uncertainties in atmospheric and aerodynamic models were considered, highlighting the need for the effect of both epistemic and aleatory uncertainties to be considered from the very beginning of the design phase in order to gain an estimation of the impact on the performance of the vehicle. Integrating the trajectory using a static, nominal optimal control law with randomly perturbed models allows for a sensitivity analysis of the obtained control law and the deviation of the results from the target final conditions to be evaluated. A second multiple optimisation approach is then performed, giving a performance expectancy curve based on the objective function, e.g., the final mass, which can be used at the preliminary stage of the vehicle design to estimate the performance of the vehicle while accounting for uncertainties in the simulation models.

Future work will aim towards the implementation of further tools for the robust optimisation of transatmospheric trajectories, with the aim to optimise the expected values of performance while minimising the effect of uncertainties. The authors are currently working on a more efficient clustering technique for the mesh required by the FET method, and the identification of suitable methods and approaches which will be able to solve large scale problems that are affected by noise and that are heavily constrained. Efficient sampling techniques are also being developed that, by reducing the noise, would allow the current hybrid optimisation tool to be applied to robust optimisation. 


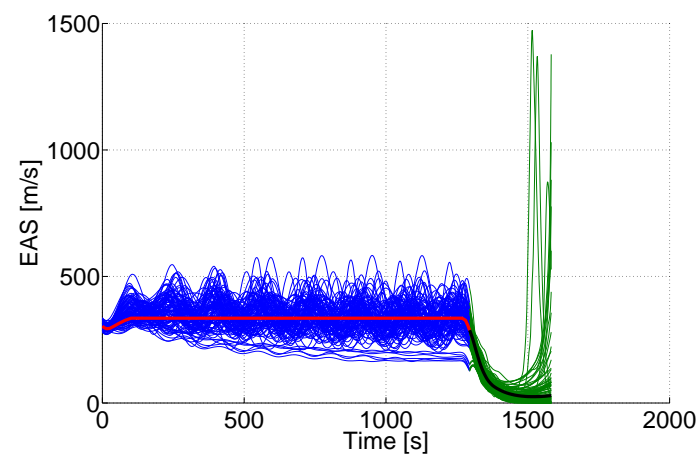

(a) Equivalent air speed

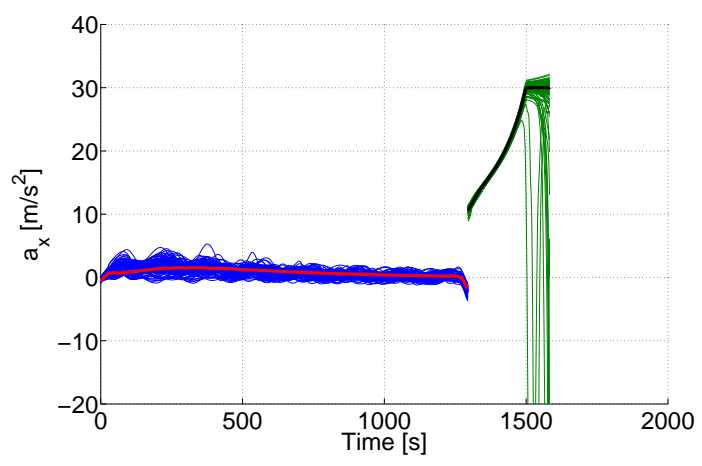

(b) Axial acceleration

Figure 7: Time evolution of the equivalent air speed (EAS) and axial acceleration $a_{x}$, where the bolded red and black lines refer to air-breathing and rocket phases respectively of the nominal trajectory, and the blue and green lines refer to air-breathing and rocket phases integrated with perturbations.

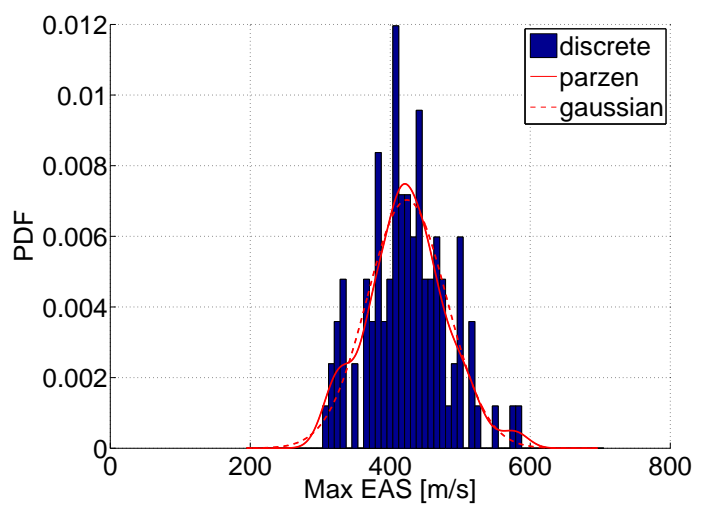

(a) Maximum equivalent air speed

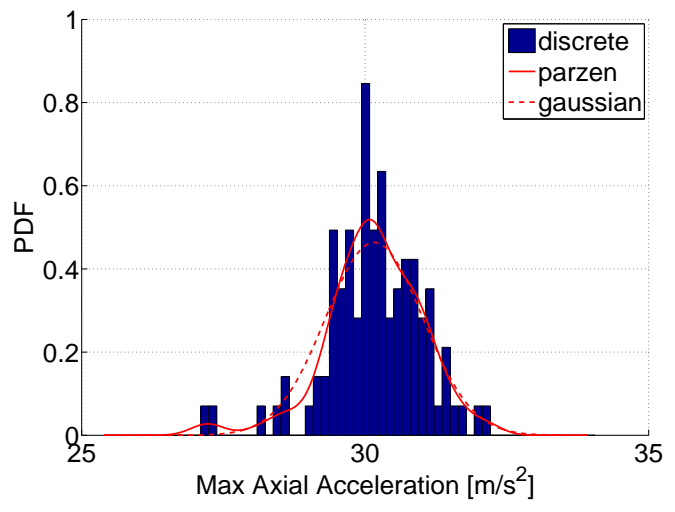

(b) Maximum axial acceleration

Figure 8: PDF of constrained values when the nominal control laws are integrated by means of perturbed atmospheric and aerodynamic models.

\section{References}

${ }^{1}$ Varvill, R. and Bond, A., "The SKYLON Spaceplane," Journal of the British Interplanetary Society, Vol. 57, 2004, pp. 22-32.

${ }^{2}$ Varvill, R. and Bond, A., "The SKYLON Spaceplane: Progess to Realisation," Journal of the British Interplanetary Society, Vol. 61, 2008, pp. 412-418.

${ }^{3}$ Butrica, A., Single Stage to Orbit: Politics, Space Technology, and the Quest for Reusable Rocketry, New Series in NASA History, The Johns Hopkins University Press, 2003.

${ }^{4}$ Betts, J. T., Practical Methods for Optimal Control and Estimation Using Nonlinear Programming, Second Edition, SIAM, 2010

${ }^{5}$ Nocedal, J. and Wright, S., Numerical Optimization, Springer-Verlag GmbH, 2nd ed., 2006.

${ }^{6}$ Costa, M. and Minisci, E., "MOPED: a Multi-Objective Parzen-based Estimation of Distribution algorithm," Evolutionary Multi-Criterion Optimization. Second International Conference, EMO 2003, edited by C. Fonseca, P. Fleming, E. Zitzler, K. Deb, and L. Thiele, Vol. 2632 of LNCS, Springer, Faro, Portugal, 08-11 April 2003, pp. 282-294.

${ }^{7}$ Vasile, M., Minisci, E., and Locatelli, M., "An Inflationary Differential Evolution Algorithm for Space Trajectory Optimization," IEEE Transactions on Evolutionary Computation, Vol. 15, No. 2, 2011, pp. 267-281.

${ }^{8}$ Minisci, E., Campobasso, M. S., and Vasile, M., "Robust Aerodynamic Design of Variable Speed Wind Turbine Rotors," Accepted for ASME Turbo Expo 2012, Copenhagen, Denmark, June 11-15 2012. 


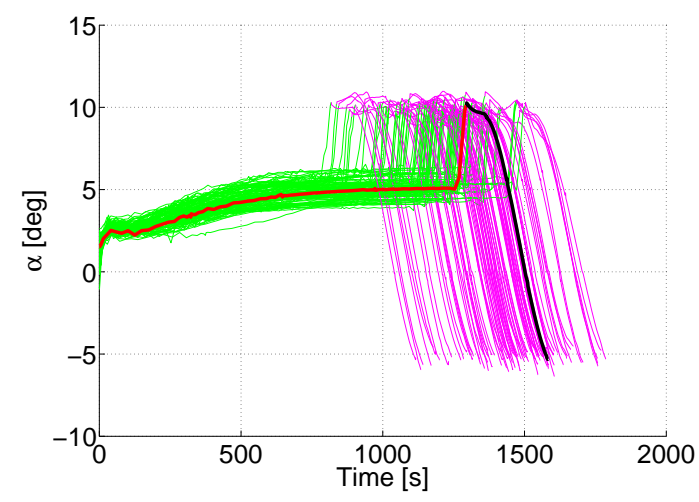

(a) Angle of attack

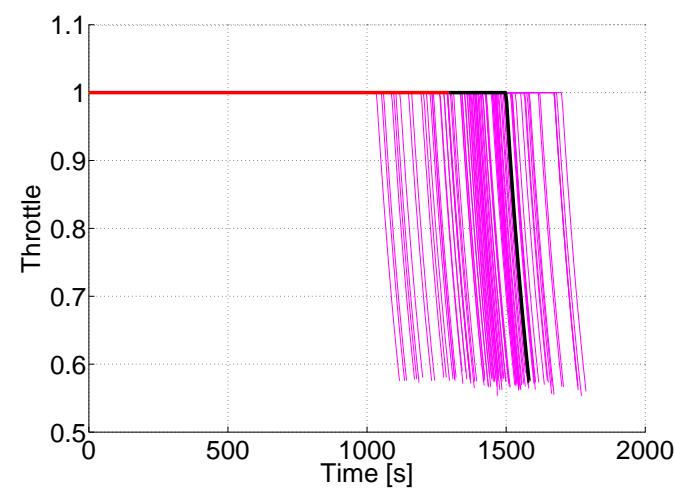

(b) Throttle setting

Figure 9: Optimized control laws where the bolded red and black lines refer to air-breathing and rocket phases respectively of the nominal trajectory, and the blue and green lines refer to air-breathing and rocket phases integrated with perturbations.

${ }^{9}$ Lozano, J. A., Larranaga, P., and Inza, I., Towards a New Evolutionary Computation: Advances on Estimation of Distribution Algorithms (Studies in Fuzziness and Soft Computing), Springer, February 2006.

${ }^{10}$ Fukunaga, K., Introduction to statistical pattern recognition, Academic Press, 1972.

${ }^{11}$ Avanzini, G., Biamonti, D., and Minisci, E., "Minimum-Fuel/Minimum-Time Maneuvers of Formation Flying Satellites," Astrodynamics Specialist Conference, AAS 03-654, Big Sky, Montana, 03-07 August 2003.

${ }^{12}$ Deb, K., Pratap, A., Agarwal, S., and Meyarivan, T., "A Fast and Elitist Multiobjective Genetic Algorithm: NSGA-II," IEEE Transaction on Evolutionary Computation, Vol. 6, No. 2, april 2002, pp. 182-197.

${ }^{13}$ Price, K., Storn, R., and Lampinen, J., Differential Evolution: A Practical Approach to Global Optimization, Springer, 2005.

${ }^{14}$ Leary, R., "Global optimization on funneling landscapes," Journal of Global Optimization, Vol. 18, No. 4, 2000, pp. 367383.

${ }^{15}$ Vasile, M., "Finite elements in time: a direct transcription method for optimal control problems," AIAA/AAS Astrodynamics Specialist Conference, AIAA Paper 2010-8275, Toronto, Canada, August 2-5 2010.

${ }^{16}$ Miele, A., Optimal trajectories of aircraft and spacecraft, No. 19910001655, JPL-956415; NAG1-516 in AGARD, Aircraft Trajectories: Computation, Prediction, Control, NASA, 1990.

${ }^{17}$ Anderson, J. D., editor, Fundamentals of Aerodynamics, McGraw-Hill Higher Education, 3rd ed., 2001.

${ }^{18}$ AIAA Guide to Reference and Standard Atmosphere Models (G-003C-2010e), 2010. 


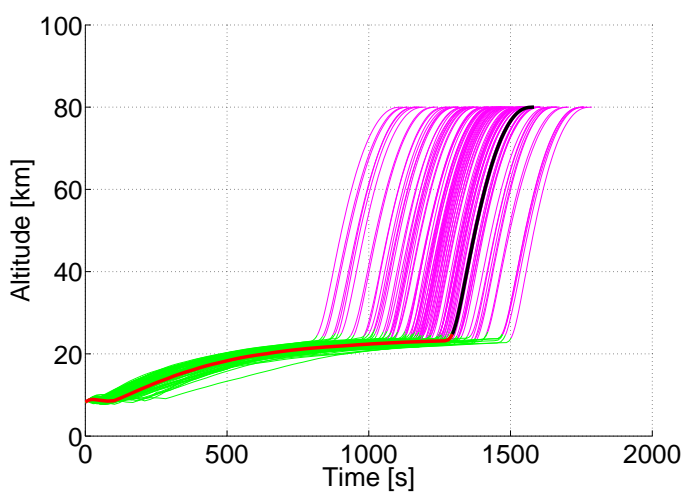

(a) Altitude

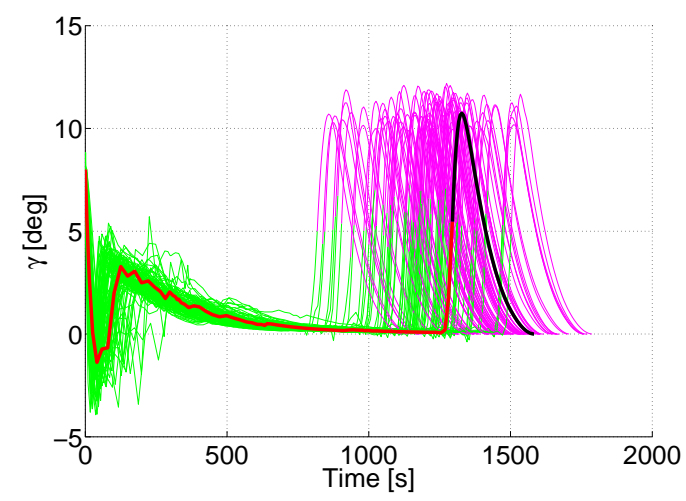

(c) Flight path angle

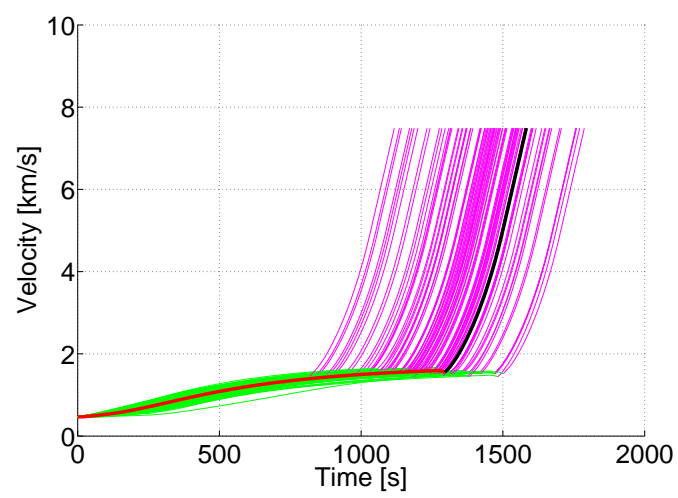

(b) Velocity

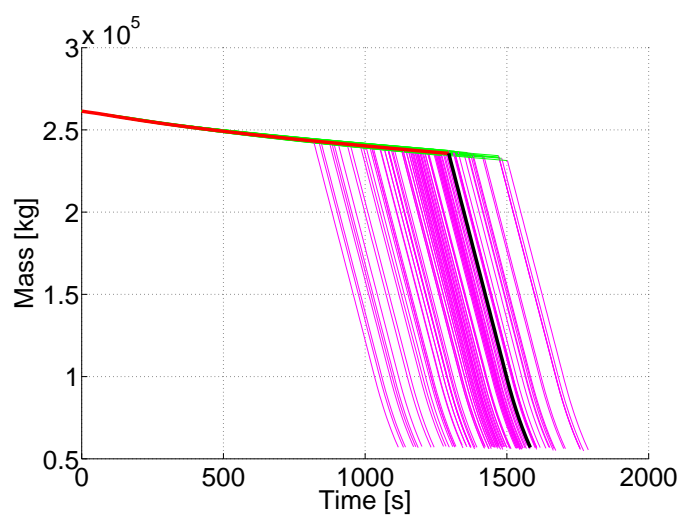

(d) Mass

Figure 10: Evolution of the states for optimized control law where the bolded red and black lines refer to air-breathing and rocket phases respectively of nominal trajectory, and the green and magenta lines refer to optimised air-breathing and rocket phases with perturbations.

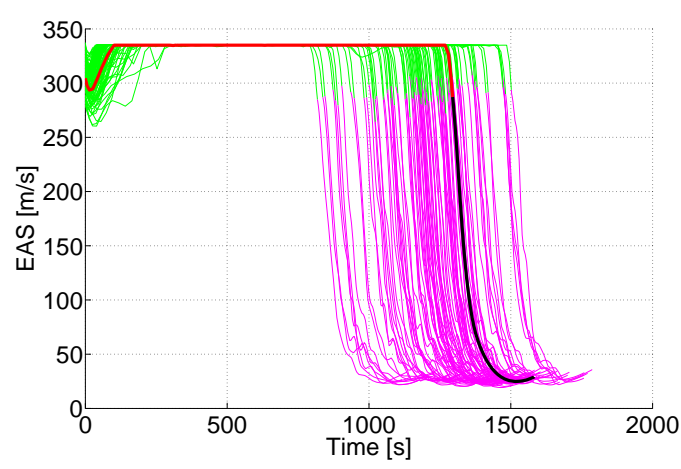

(a) Equivalent air speed

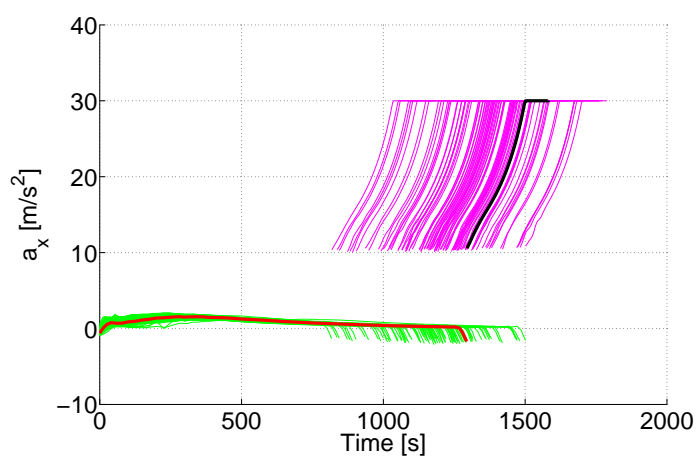

(b) Axial acceleration

Figure 11: Evolution in time of equivalent air speed and axial acceleration, where the bolded red and black lines refer to air-breathing and rocket phases respectively of nominal trajectory, and the green and magenta lines refer to optimised air-breathing and rocket phases with perturbations. 


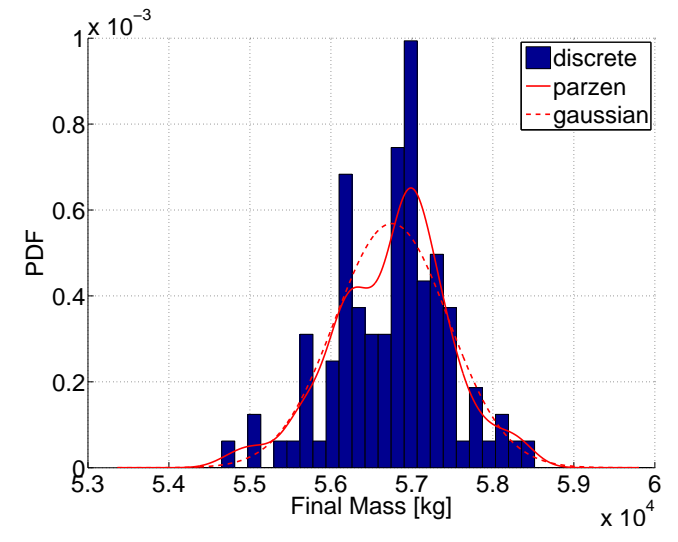

Figure 12: PDF of final mass as obtained by optimized trajectories.

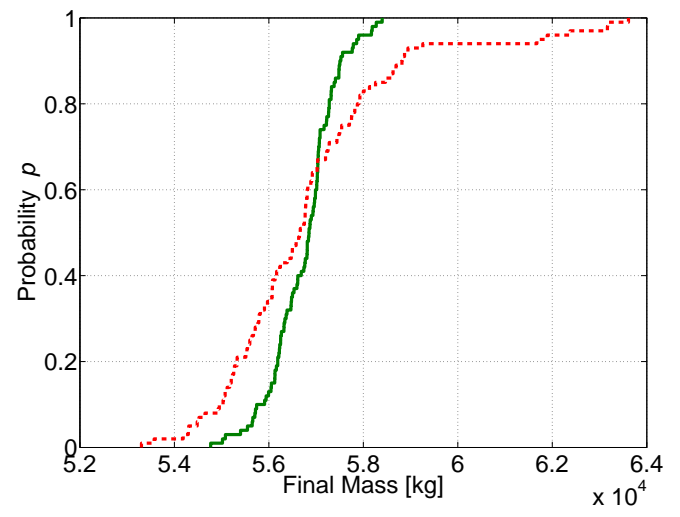

Figure 13: Comparison of CDF curves of final masses for the two approaches where the green solid curve shows the optimized trajectories and red dashed curve, those integrated with perturbations. 